\title{
Pattern-Based Competence Management: On the Gap between Intentions and Reality
}

\author{
Ralf Klamma and Zinayida Petrushyna \\ Information Systems and Databases, RWTH Aachen University, \\ Ahornstrasse 55, 52056, Aachen, Germany \\ \{klamma, petrushyna\}@dbis.rwth-aachen. de
}

\begin{abstract}
Competence management in collaborative networks depends on the identification of gaps between the actual performance and the desired behavior. Therefore, collaborative networks need means to express their goals and intentions as well as to measure this against their own performance. In this paper, we present the $i *$-based modeling framework for agent-oriented elicitation of dependencies and goal-orientation in collaborative networks. Moreover, we sketch a method to translate the resulting models into a pattern-oriented analysis framework, which allows to compare the models with social network analysis results on real media traces left by the networks while collaborating using social software. This results in the identification of hidden or missing competences within a network. We will illustrate the approach with examples from ongoing project work. Finally, we discuss a collection of collaborative patterns indicating which (meta-)competences can be acquired.
\end{abstract}

Keywords: competence, collaborative networks, agents, i* modeling, social network analysis, patterns.

\section{Introduction}

The gap between reality and intentions points out what fails in the reality to come to the intention state. Competences fulfill the goals by performing actions with the purpose to achieve results. As soon as we identify the gap, we can define what actions should be performed to achieve the goal, thus we can say what competences fails and should be applied. The question we aim to answer in this paper is how to generate models of collaborative networks that reflect intentions and reality.

In the next section we describe how competences, competence management (CM) and development are defined in the scope of this paper. Afterward, we explain what are the patterns appropriate for and what social network analysis patterns can reveal. In Section 4 we introduce the basics of Actor Network Theory, Community of Practice $(\mathrm{CoP})$ and we precisely describe agent-based $i^{*}$ modeling framework. In Section 5 we describe the model of one of test-bed scenarios representing collaborative networks in a university. Later we combine the model with the pattern oriented framework. Thus we extend the model with patterns extracted using social network analysis and present some examples of competences management and development that can be draw out from the model. The paper is finished with discussions where we reveal some related works and conclusions. 


\section{Preliminaries}

Before we start to model collaborative networks $(\mathrm{CN})$ and consider competences in models, we introduce some terms important in this work. The term competence stems from Latin competentia that means agreement or conjunction. Actual definition of competence is often used within working associations to form quality standards [3].

Although numerous definitions for competence exist, many scholars agree that the term is associated with skills and abilities that people currently hold and may acquire in the future [10]. Competences allow an individual to perform an organizational or a personal task. For example, social competences like socializing, team working and social thinking are abilities that a manager should have.

$C M$ stands for the identification of competences and the optimization in fitting persons with according competence profiles to appropriate tasks. Competence development and learning or advanced training can be used synonymously as it is connected with the process of knowledge acquisition. The development of competences is based on extending or acquiring expertise.

\section{Management and Development of Competences Using SNA Patterns}

Both, management and development of competences depend on the actual status of competences in an environment, which we can extract by precisely analyzing the environment. The activities, that repeat periodically in the environment, e.g., people wake-up, eat, work, have meetings and reports, are called patterns. Pattern analysis bears on the real situation of the environment. It can advise what competences are presented or needed currently in the environment.

Pattern analysis was firstly introduced by [1]. A pattern consists of a description of a problem, a context where it is applied, forces, which are influencing appearance of the problem, and possible solutions to the problem Moreover patterns can be stimulations to change the current situation [11]. The purpose of patterns is not only to analyze the reality but as well to give advice or enable people to figure out how to proceed in the current situation. We choose social network analysis (SNA) to identify patterns of CNs.

\subsection{SNA Applications}

SNA technique reveals hidden roles and goals of workers, which are not highlighted by applying other analysis [9]. Above all, the characteristics of networks and their nodes indicate persons with the biggest and smallest social capital and the highest reputation. It has been proved that the centrality of a node in a student network influences onto students' performance, team outcomes and satisfaction from learning [2]. Moreover, using SNA one can calculate the frequency of collaborations and consensus degree in a network.

In any case, the information SNA gives us must be interpreted and further steps must be inferred. If a network is not dense enough and clusters of the network do not communicate with each other, this can be a reason why the network is not productive 
enough because teams (clusters) are not communicating with each other or only through one channel. We name the pattern for the described problem silent collaboration. It is clear that in the case of silent collaboration no or few team members possess social competences. Thus, a competence manager infers why the productivity is not high and can enhance the situation by organizing an event where isolated teams get to know each other.

\subsection{Pattern Analysis Oriented Framework}

In our past work we created a framework that uses SNA technique for discovering patterns in digital media communities [7]. We defined the rules of communications within CNs and the spammer, the troll, the answering person, the questioner, the communicator, and some other patterns were defined using the rules. For example, the troll is a person who interacts within a thread/topic that he was initiating and there are no more threads/topics where the troll contributes to but not a thread/topic initiator.

In our experiments with communities in digital media, mailing lists in our case, we defined the following patterns: unluckily initiators, reply senders, reply receivers, communicators and cross-users. We notice that in threads with many reply senders (the outcoming degree of links is higher than average), which are answering the questions, posemo ${ }^{1}$ and negemo ${ }^{2}$ words are used. In such a case we can infer that people have longtime or personal contact with each other and can freely express themselves.

\subsection{Contribution to Competence Management}

The application of SNA contributes to evaluation of competences of CNs members as the rating of social skills is possible using SNA measures. Considering activities of the members in the past and evolution of their positions in CNs we can find which activities infer which competences. Scrutinizing goals of CNs and their members, the appropriate activities can be proposed for acquiring required competences.

\section{Agent-Based Modeling of Collaborative Networks}

\subsection{Agents of Collaborative Networks}

Before retrieving patterns of CNs we want to conceptualize the structure of the networks and their environments. That is why we refer to Actor Network Theory (ANT) presented by [4]. He claims that any object in a system is an actor, be it a human or not. Therefore, a system is represented as a set of actors that are depending on each other. Further, we consider a $\mathrm{CN}$ as a network of actors connected to each other thus depend on each other.

Figure 1 depicts the basic idea of Actor Network. Any thing is an Actor. In previous work [6] we defined that the Actor Network of CNs in digital environments includes Process, Media, Community, Artefact, and Agent actors.

\footnotetext{
${ }^{1}$ Agree, improve, support.

${ }^{2}$ Contend, protest, decline.
} 


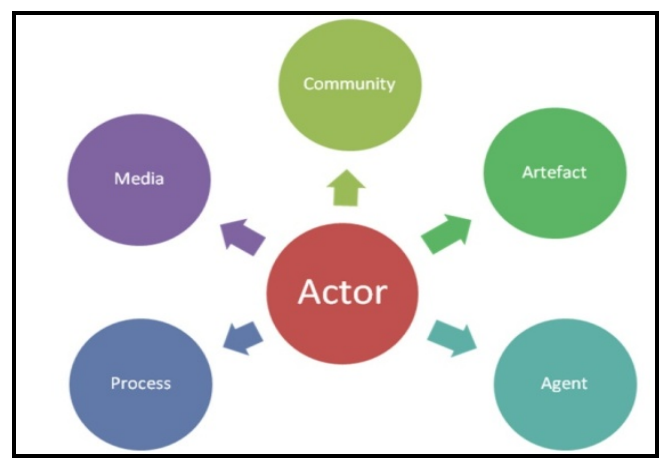

Fig. 1. Actors of digital collaborative network

We consider digital environments as Media actors, e.g., a digital learning environment, forums, virtual classrooms. Artefact actors are bits of Media, e.g., a course, a forum post, a video lecture. Agent actors correspond to persons in a CN. Community actors are sets of Agent actors that collaborate using Media and communicate operating Artefact actors. Processes are mostly initiated by actor's activities, e.g., using Media and operating Artefact actors.

Latour emphasizes that any actor is influenced by another one. Thus, we need to define the dependencies of actors. That is why we appeal to sociology and consider Communities of Practice.

\subsection{Community of Practice}

The concept of CoP was precisely described by Wenger [12]. The author postulates that some communities are organized because the members of the communities are interested or evolved in the same practices. Moreover, Wenger states that a CoP has three dimensions that define it. These are joint enterprises (JE), mutual engagement (ME) and shared repertoire (SR). JEss are policies and rules which all members follow, MEs correspond to members' collaborations and the SR is the knowledge repository formed by information possessed by a community.

Considering the described dimensions we can determine how Actors depend on each other: communities depend on Media as the latter is liable to provide an environment for collaborations (ME); Media and Artefacts depend on Processes as without Processes it is impossible to create, extend or change Artefacts and attach them to Media (JE); Processes depend on Communities and Agents, which initiate activities (JE); Agent depends on Communities as the latter identifies the role of Agent in Community based on his knowledge and his collaborations (SR, ME). We can mention some more of the possible dependencies but abstain from it for the sake of brevity.

Considering dimensions of $\mathrm{CoP}$ and agent-based nature of $\mathrm{CNs}$ we can conceptualize $\mathrm{CNs}$ in general and adopt the model of $\mathrm{CNs}$ to our test-bed scenarios. An appropriate modeling technique that satisfies our requirements must be found. First of all, the technique must be agent-oriented, as we consider a $\mathrm{CN}$ as a set of agents. Secondly, it must be possible to explicitly define dependencies between actors. Thirdly, we require the consideration of social characteristics to discover competences of CNs. 
Finally, models must be extendable so that we can include facts from reality in the form of patterns.

\section{3 i* Agent-Oriented Modeling Technique}

The $\mathrm{i}^{*}$ language is an agent-oriented high-level modeling approach [13]. It was developed for the design of business processes on a strategic level. The core of $\mathrm{i}^{*}$ models are motives, interests, options and constraints of agents in a business process. Cooperation among agents is needed for fulfillment of common goals. Due to dependencies between goals, tasks, resources and the agents themselves, agents become also vulnerable. In $i^{*}$ we find the following premises: agents act intentionally, because they are following their goals, have beliefs, competences, commitments, needs and desires. Agents are strategic actors, because they have to proceed according to their plans. Intentional dependencies can be made explicit with $i^{*}$ to disclose the reasons behind observable processes. Questions to an $i^{*}$ model are: Who is deciding what? Which options does an agent have? How can I reduce critical dependencies?

In $i^{*}$ we use the strategic-dependency-model (SDM) with a focus on the intentions of agents so that it perfectly fits the requirements of communities. It serves as an abstract view on the external relations of an agent. It delivers very concrete models, e.g., if information is delivered from agent A to agent B but never the other way around, agent $\mathrm{B}$ is depending on agent $\mathrm{A}$ but not vice versa.

Here we present a short description of conceptual modeling components in $\mathrm{i}^{*}$ and use it later in Figure 2 and 3 A so-called dependee is an agent that influences other agents' performance, e.g., Website of the course in Figure 5.1. The depending agents are dependers, e.g., Learner. The object through which the dependency is defined is called dependum, e.g., announcements. The dependencies can be one of the following four types - resource dependency, task dependency, goal dependency and softgoal dependency. A resource dependency like announcements exists in case a target Website of the course provides a resource to a source Learner. There is a task dependency such as create/find/reuse when a source Artefact expects a target Learner to execute a task and goal dependency indicates the case when a source Artefact expects that a target Teacher achieves a goal, e.g., Evaluation. The softgoal dependency like to focus on professional competences in Figure 5.2 is used when the goal cannot be described clearly, although a dependency exists.
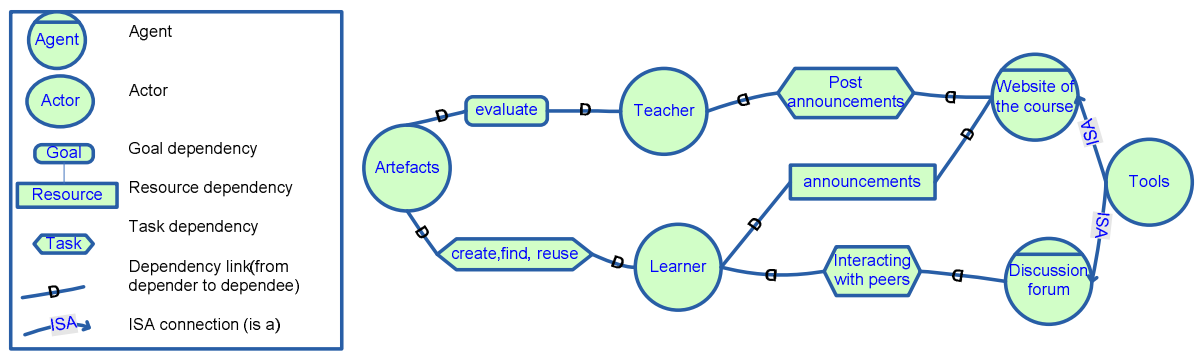

Fig. 2. Model of a collaborative network within a university course 
Although $\mathrm{i}^{*}$ is already helpful, it does not solve the problem how to manage competences in CNs. We need to extend it for accomplishing our needs. We propose to combine the modeling framework with the SNA pattern-based framework (PBF) and describe all the working procedures of the SNA PBF. On the preparation phase of the framework, we examine CNs and model them. Based on SNA patterns, models can be extended with additional information. Then the gap between imagined scenario how it is intended to be and real scenario how it is in reality can be filled, considerately existed and missing competences of CNs are defined.

In the following, we present the outcome of our SNA PBF for CM cooperating with $\mathrm{i}^{*}$ modeling framework. The presented scenario is taken from a real university course of one of the test-bed partners in EU IP ROLE.

\section{University Collaboration Scenario}

Courses in universities are constructed in such way that they require a lot of team work. Universities pursuit of letting students to acquire both professional and social competences. On the Figure 2 we consider the university course that utilizes Web technologies for some of its tasks. It provides possibilities for collaborations and awareness, such as Discussion forum and Website of the course.

In order to apply the methods of the SNA PBF we need to define collaboration networks that exist in the scenario. There are at least two types of CNs possible: Learner-Learner CNs and Learner-Teacher CNs. With the help of the framework we can identify a learner network characteristics in Learner-Learner CNs, in LearnerTeacher $\mathrm{CN}$ and a teacher network characteristics in Learner-Teacher CN. Based on numerous network characteristics it is possible to answer whether the learner's status in Learner-Teacher or the status in Learner-Learner CNs correlate with the learner's marks. As a result it can be discovered that the learner is too communicative (degree/betweenness/closeness centralities of the learner) but pays not enough attention to managing learning material (create/find/reuse task for Artefact).

The person responsible for CM and development (the course manager or the teacher of the course) can help the learner to focus on professional competences so that the learner can better succeed in the course. The learner can be asked to handle more often with Artefacts, e.g., to create a knowledge map about a topic from the

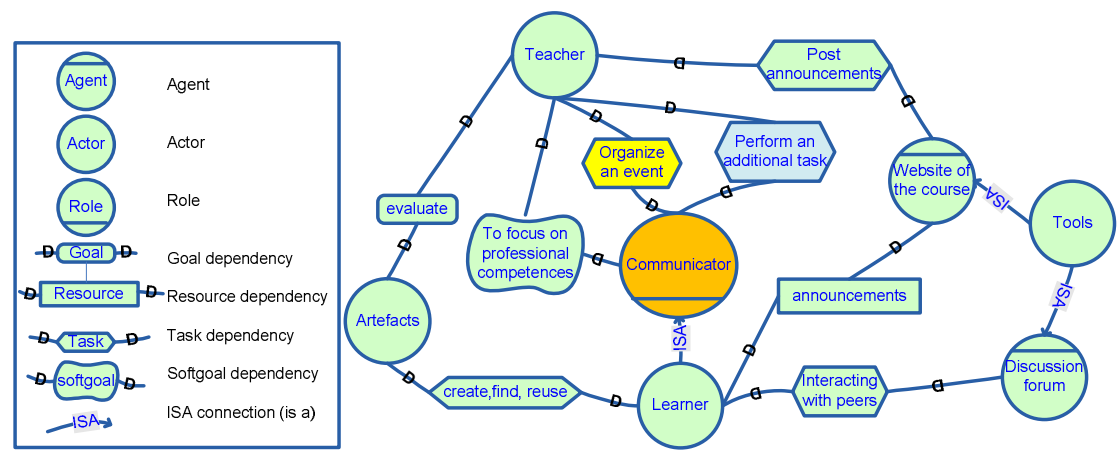

Fig. 3. Model of a CN using i*modeling framework and SNA pattern-oriented framework 
course. Afterwards, the learner can discuss his Artefact in a Discussion Forum as according to the communicator pattern, it is the part of learning he enjoys. While this is a competence development application, the next scenario is an example of CM.

On the other hand, if the teacher of the course needs to organize an event for the students of the course, the learner is the right person for the task.

The result of the application of the SNA PBF is depicted in Figure 3 Here we use the Role $\mathrm{i}^{*}$ notation for illustrating the communicator pattern.

\section{Related Work}

The $i^{*}$ modeling framework we used here proposes an ostensive type of modeling as the framework aims to express the goals the agents of the systems want to achieve. Another type of modeling is performative modeling, which depicts collaborations as it happens in reality (Feldman and Pentland, 2003). Even goal-oriented modeling (i*) describes how collaborations are expected to be, the combination of $i^{*}$ modeling with the pattern-oriented framework makes the models both reflect the reality of a $\mathrm{CN}$ and the goals of actors, the intensions. However, the latest elaborated survey on modeling [9] distinguishes only between ostensive and performative modeling techniques ${ }^{3}$ for collaborative technologies.

The model of collaborations using the concept of patterns was recently published by [8]. The work describes capturing and reusing recurring segments of work or parts of collaborations. Patterns are identified through analysis of actions of users in a system.

Another approach for CM applies a competence performance approach [5]. The authors collect information about competences needed for task performance and based on the information and competence profiles of workers appropriate tasks are assigned to suitable workers. If workers do not have required competences appropriate courses are assigned to workers.

\section{Conclusion}

We introduced the notions of patterns and competences and showed how competences can be managed and developed using patterns. We particularly consider social network analysis techniques to identify patterns. Moreover, we applied the $i^{*}$ modeling technique that allows us to conceptualize one of our test-bed scenarios of CNs. We considered Actor Network Theory and Communities of Practice in our model. Finally, we propose the SNA pattern based framework that extend the goal-oriented $i^{*}$ model with facts from reality extracted using SNA patterns. We show how such models support competence development and management tasks.

Acknowledgments. The research leading to these results has received funding from the European Community's Seventh Framework Programme (FP7/2007-2013) under grant agreement no 231396 (ROLE project).

${ }^{3}$ UML models, workflow, coordination theory, Grounded theory, temporal models (rhytms), social network models or language action perspective. 


\section{References}

1. Alexander, C.: A Pattern Language: Towns, Buildings, Construction. Center for Environmental Structure Series. Oxford University Press, New York (1978)

2. Baldwin, T.T., Bedell, M.D., Johnson, J.L.: The Social Fabric of a Team-Based M.B.A. Program: Network Effects on Student Satisfaction and Performance. The Academy of Management Journal 40(6), 1369-1397 (1997)

3. Cheetham, G., Chivers, G.E.: Professions, competence and informal learning. Edward Elgar Pub. (2005)

4. Latour, B.: On Recalling $\{$ ANT $\}$. In: Law, J., Hassard, J. (eds.) Actor-Network Theory and After, Oxford, pp. 15-25 (1999)

5. Ley, T., Lindstaedt, S., Albert, D.: Supporting Competency Development in Informal Workplace Learning. In: Althoff, K.-D., Dengel, A.R., Bergmann, R., Nick, M., RothBerghofer, T.R. (eds.) PWM 2005. LNCS (LNAI), vol. 3782, pp. 189-202. Springer, Heidelberg (2005)

6. Klamma, R., Petrushyna, Z.: The Troll Under the Bridge: Data Management for Huge Web Science Mediabases. In: Proc. Informatik 2008 Band 2 - Workshop Digital Social Networks, pp. 923-928. Gesellschaft für Informatik (2008)

7. Klamma, R., Spaniol, M., Denev, D.: \{PALADIN\}: A Pattern Based Approach to Knowledge Discovery in Digital Social Networks. In: Tochtermann, K., Maurer, H. (eds.) Proceedings of I-KNOW 2006, 6th International Conference on Knowledge Management, Graz, Austria, September 6 - 8, pp. 457-464. Springer, Heidelberg (2006); J.UCS (Journal of Universal Computer Science)

8. Papageorgiou, N., Verginadis, Y., Apostolou, D., Mentzas, G.: A collaboration pattern model for virtual organizations. In: Leveraging Knowledge for Innovation in Collaborative Networks. IFIP Advances in Information and Communication Technology, vol. 307, pp. 61-68. Springer, Heidelberg (2009)

9. Poltrock, S.E., Handel, M.: Modeling Collaborative Behavior: Foundations for Collaboration Technologies. In: HICSS, pp. 1-10. IEEE Computer Society, Los Alamitos (2009)

10. Sampson, D., Fytros, D.: Handbook on Information Technologies for Education and Training, Berlin, Heidelberg, pp. 155-177 (2008)

11. Schuler, D.: Liberating Voices: A Pattern Language for Communication Revolution, p. 504. MIT Press, Cambridge (2008)

12. Wenger, E.: Communities of Practice: Learning, Meaning, and Identity. Cambridge University Press, Cambridge (1998)

13. Yu, E.S., Mylopoulos, J.: Towards Modelling Strategic Actor Relationships for Information Systems Development - With Examples from Business Process Reengineering. In: Proceedings of the 4th Workshop on Information Technologies and Systems, Vancouver, B.C., Canada, December 17-18, pp. 21-28 (1994) 